\title{
Challenges When Engaging Diabetic Patients and Their Clinicians in Using E-Health Technologies to Improve Clinical Outcomes
}

\author{
Brian Edward Dixon ${ }^{1,2,3}$, Abdulrahman Mohammed Jabour ${ }^{1}$, \\ Erin O'Kelly Phillips ${ }^{4}$, and David G. Marrero ${ }^{4,5}$ \\ ${ }^{1}$ Department of BioHealth Informatics, School of Informatics and Computing, \\ Indiana University, Indianapolis, IN, USA \\ \{bedixon, ajabour\} @iupui.edu \\ ${ }^{2}$ Center for Biomedical Informatics, Regenstrief Institute, Indianapolis, IN, USA \\ ${ }^{3}$ Center for Health Information and Communications, Department of Veterans Affairs, \\ Veterans Health Administration, Health Services Research and Development Service, \\ Indianapolis, IN, USA \\ ${ }^{4}$ Diabetes Translational Research Center, School of Medicine, Indiana University, \\ Indianapolis, IN, USA \\ \{ekokelly, dgmarrer\}@iu.edu \\ ${ }^{5}$ Department of Endocrinology, School of Medicine, Indiana University, \\ Indianapolis, IN, USA
}

\begin{abstract}
Diabetes mellitus (DM) is a chronic disease affecting more than 285 people worldwide and the fourth leading cause of death. Increasing evidence suggests that many DM patients have poor adherence with prescribed medication therapies, impacting clinical outcomes. Patients' barriers to medication adherence and the extent to which barriers contribute to poor outcomes, however, are not routinely assessed. We designed a dashboard for an electronic health record system to integrate DM disease and medication data, including patientreported barriers to adherence. The dashboard was pilot tested at multiple ambulatory clinics to examine whether integrated electronic tools can support patient-centered decision-making processes involving complex medication regimens for DM and other chronic diseases. During pilot testing, we encountered several challenges when engaging patients and clinicians in using the dashboard as well as a portal used to gather self-reported psychosocial information directly from patients. In this paper we explore those challenges and suggest methods for better supporting the adoption and use of e-health technologies to improve care delivery processes as well as health outcomes for populations like diabetic patients.
\end{abstract}

Keywords: Medication Adherence, Type 2 Diabetes Mellitus; Computerized Medical Records Systems, Personal Health Records, Physician-Patient Relations, Drug Monitoring, Patient-Centered Care. 


\section{Introduction}

Diabetes mellitus (DM) is a chronic disease affecting more than 285 people worldwide and the fourth leading cause of death. Globally the prevalence of DM continues to rise at nearly epidemic rates, driven by urbanization, growing increases in obesity, and aging of populations [1]. Findings from several studies investigating the quality of DM care reveal a discrepancy between system-level disease management strategies and outcomes [2-6]. In essence, even though there are improved treatment strategies, expected outcomes are not occurring at a commensurate level. Therefore greater emphases on patient-level factors that may explain DM intervention outcomes are being explored.

One such factor is adherence to complex medication regimens. Increasing evidence suggests that patients with diabetes often have poor adherence with prescribed medication therapies [7, 8]. However, the reasons why patients do not take their medications as prescribed are poorly understood [9]. Previous studies on adherence have relied on patient self-report data on medication use, or they have used more "objective" measures such as gaps in prescription coverage or technologies to determine if patients are taking their medications. An example is electronic MEMS caps, which document when a pill bottle is opened [10-12]. All of these approaches have limitations which include accuracy; reporting and response bias; or limited effect size [13].

Despite existing evidence and efforts, patient-reported barriers to medication adherence and the extent to which those barriers contribute to poor DM outcomes are not currently assessed routinely in clinical practice [14]. Indeed, few have assessed the role of barriers perceived by patients to medications use and how perceived barriers may be addressed by intervention.

To address medication adherence issues facing individuals with complex medication regimens, we developed a clinical information system to electronically integrate the capture and presentation of information regarding DM patients' disease management, medication adherence, and perceived barriers to adherence [15]. The system combines objective data regarding medication possession ratios with laboratory and point-of-care testing data as well as patient-entered data on perceived barriers to adherence. By routinely capturing patient-reported barriers and integrating such information at the point-of-care with other electronic health data, we seek to better inform DM therapy decision-making processes.

In this paper, we describe the system and our experience pilot testing the system in three primary care clinics. During the pilot testing, we encountered several challenges when engaging patients and clinicians in using both the dashboard as well as a portal designed to gather patient-reported barriers to medication adherence. Here we discuss those challenges and suggest methods for better supporting the adoption and use of e-health technologies to improve care delivery processes as well as health outcomes for populations like DM patients. 


\section{Methods}

\subsection{System Description}

The primary system of interest is a dashboard (Figure-1) used by clinicians (e.g., physicians, nurses, pharmacists) in the context of routine primary care. Clinicians review the information on the dashboard either before or during a patient visit to understand the patient's current adherence to their medication regimen for DM2.

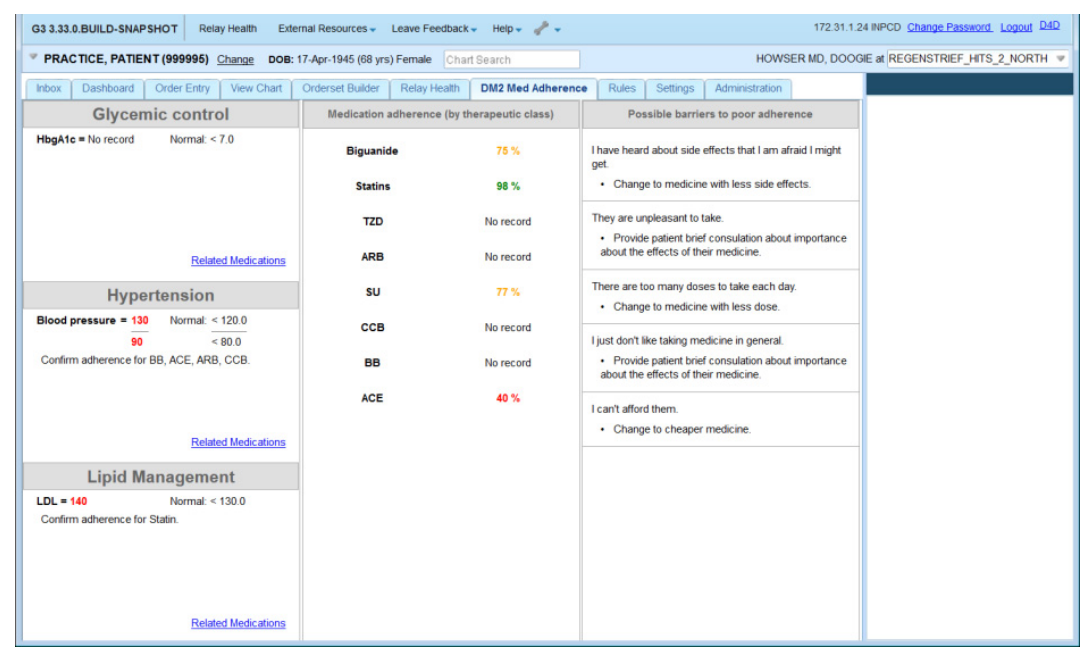

Fig. 1. Screenshot of clinical dashboard designed to integrate medication adherence information from multiple sources into electronic health record (EHR) system

The clinical dashboard is a Java-based module designed to plug into the Regenstrief CareWeb ${ }^{\mathrm{TM}}$ framework, an open-source electronic health record (EHR) platform developed by the Regenstrief Institute's Center for Biomedical Informatics. CareWeb is a web-based version of the Regenstrief Medical Record System (RMRS) [16], providing primary care clinicians in Eskenazi Health facilities access to patients' medical records. The framework provides all plugins with a common data retrieval and display interface, which the dashboard uses to access and visualize patient information. The framework also allows the dashboard to receive notification of and react to various events, including when a patient is selected by the user.

The dashboard was explicitly designed to be passive, or non-interruptive. When the clinician selects a patient, the dashboard refreshes with content from multiple sources: the electronic health record (RMRS) providing recent physiological data, pharmacy data detailing which medications have been dispensed to the patient, and patiententered information on their personal challenges in taking their medication as prescribed. System users have the option to view the dashboard or ignore it.

When information is displayed, the dashboard uses several visual techniques to highlight normal as well as abnormal patient information. When a patient's blood pressure or glycosylated hemoglobin (HbA1c) is elevated, is it highlighted in red; 
normal values are shown in black. When the patient is believed, based on pharmacy claims information, to be non-adherent to a class of DM medications, the information is highlighted in red; acceptable levels are highlighted in green; potentially problematic values are shown in yellow. Such color differentiation enables quick review of the dashboard contents by clinician users.

Electronic Health Record Data. The left panel of the dashboard displays information extracted from the patient's EHR. Three types of EHR data are displayed: blood pressure, HbA1c, and cholesterol. These data were targeted because they are relevant to DM patient populations. Clinicians routinely measure and analyze HbAlc levels to determine how well DM patients keep their disease under control. High blood pressure and poor lipid management are also commonly co-related with DM2, leading to medications that treat multiple conditions. Therefore clinicians can conveniently review common physiologic data for DM patients in one location.

Medication Adherence Data. Adherence to DM medications and cardiovascular risk factors is displayed in the middle panel of the dashboard. The information originates from the Medication (Med) Hub, an independent web service within the Regenstrief technology infrastructure [17]. The Med Hub serves as a central source of medication data for all CareWeb applications. Two sources of information currently feed the Med Hub: the Wishard Health Services pharmacy system, an internal database of medications dispensed to Wishard patients many of whom do not have insurance, and SureScripts, a national dataset of medications dispensed from independent chain and mail-order pharmacies.

Using the pharmacy data available from the Med Hub, we calculate the proportion of days covered (PDC), a ratio representing whether the patient possessed a drug or a class of drugs (e.g., all oral DM medications) during a defined measurement period. The PDC has been shown in numerous studies to accurately identify patients who fail to fill or refill their medications as directed by their physician or pharmacist [18]. We use a dichotomized 6-month (180-day) PDC with a cut-off point of $80 \%$, which we have found to provide the strongest and most reliable correlation with patient glycemic control [19].

Patient Feedback. The right panel of the dashboard displays feedback from patients on their challenges in taking their medications. Patients interact with a personal health record (PHR) system to answer questions about their medication regimen. The data are then available for query by the DM application for display.

The PHR was developed using the Open Medical Record System (OpenMRS) platform [20], an open-source EHR that originated at Regenstrief but is now implemented and supported by a worldwide collaborative involving individuals from numerous counties involved in EHR, PHR, and m-Health initiatives [21]. Although OpenMRS was selected for our project, the dashboard could be integrated with any other PHR.

OpenMRS includes a forms module that allows collection of standardized data from patients. Using the forms module, we implemented a 5-point Likert style, 
validated questionnaire developed by researchers at the Diabetes Translational Research Center affiliated with the Indiana University School of Medicine [22, 23]. The questionnaire uses 20 items to assess possible barriers to medication adherence. For example, valid responses as to why one may not take his or her prescribed medications include "I can't afford them" and "I just forget to take them." Individual items are grouped into categories and ranked based on aggregate scores from patient responses. The highest ranking three categories are stored for retrieval by the dashboard.

The 20 items in the patient survey are factor analyzed with varimax rotation and correlated with perceived health status as well as satisfaction measures. Five factors or subscales can be identified and displayed to clinical users: poor access to medications; poor communication with providers; poor understanding of medications and/or difficulty in taking them; presence of side effects; and system-level barriers to use. Previous analysis suggests that persons with poor CVD risk factor control have more reported barriers that may inhibit medication adherence than do persons with good risk factor control [23].

\subsection{Information Flow}

Physiologic and medication adherence data are captured routinely from health care delivery processes and electronically sent to the RMRS. Patient feedback on medication adherence requires manual completion of the questionnaire within the PHR. Patients are asked every 2-3 months to complete a survey as previous studies found adherence to be fairly consistent over time.

The information needed for the dashboard is queried in parallel with other CareWeb processes when a clinician opens the electronic medical record for a patient (Figure-2). First, the CareWeb server notifies the DM2 module that a patient record has been selected. The module then, in parallel, requests the three sets of data from the EHR, PHR, and CDS web service. The EHR returns physiologic data for the selected patient. The PHR returns the highest ranking categories identified by the patient from their responses to the questionnaire. The CDS service retrieves the pharmacy data from the Med Hub service and calculates the PDC. The PDC is returned to the DM module. All three data sets are stored in the server's cache until the clinician selects the DM tab within the CareWeb application. Upon selection, the data sets are rendered into their respective columns for review by the clinician.

\subsection{System Pilot}

We completed development and implementation of the dashboard, DM module, and PHR in late 2012. In early 2013, we enrolled 15 primary care clinicians (Medical Doctors and Nurse Practitioners) at three community health centers part of Eskenazi Health, a publically funded health system that serves primarily the uninsured and underinsured population of the Indianapolis metropolitan area. Following enrollment of physicians, we enrolled 96 diabetic patients under the care of one participating clinicians. The pilot ran through the end of 2013. 


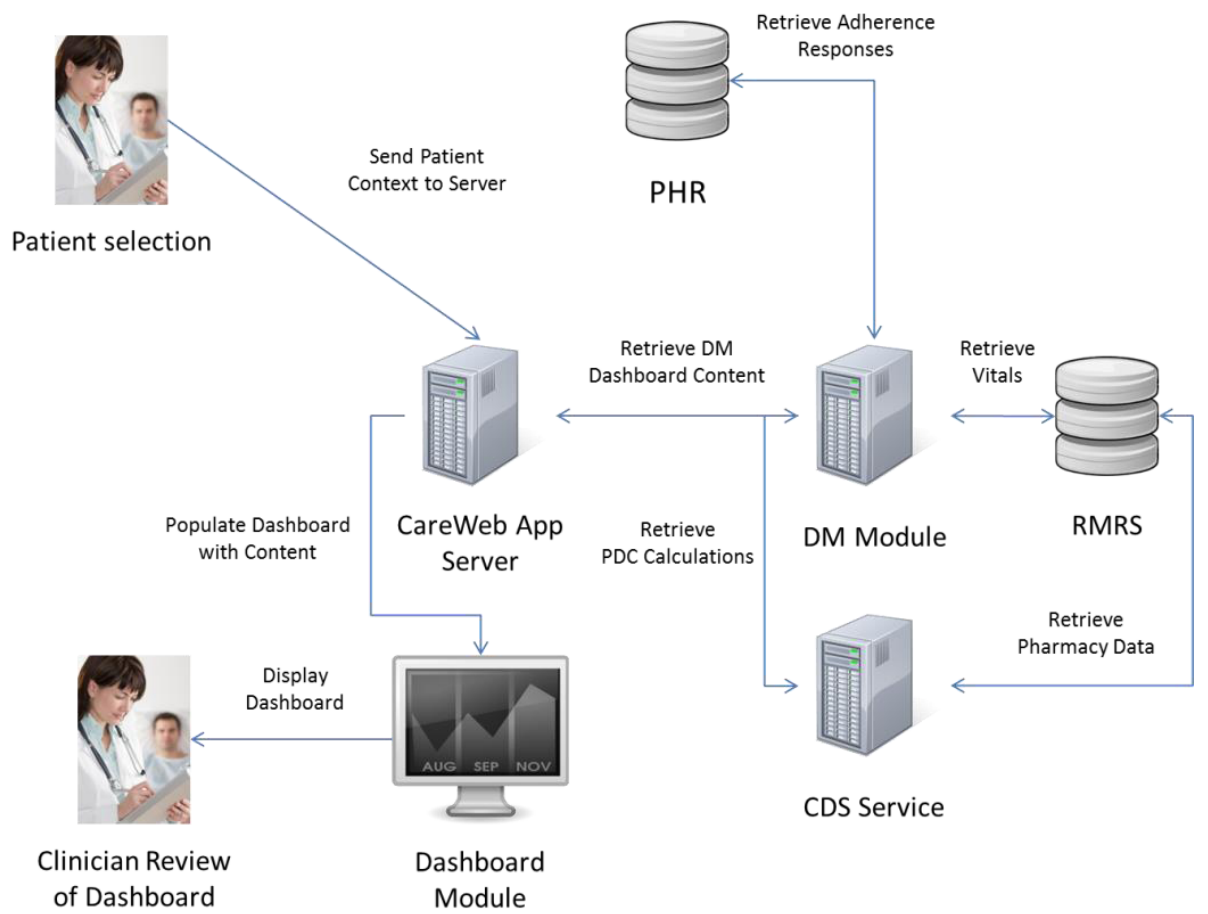

Fig. 2. Information flow diagram depicting the architecture of the DM clinical information system module and its integration with existing electronic health record, clinical decision support, and personal health record system components

\subsection{Study Design}

We collected baseline perceptions of providers regarding medication adherence discussions between them and their patients. Baseline perceptions of patients regarding current conversations occurring about medication adherence were also captured. In addition, we collected demographic information about patients and providers to enable comparison across health center locations and socioeconomic data, which has been shown to effect patient access to the Internet impacting PHR usage [24, 25].

Following enrollment, a research assistant created PHR user accounts for each of the enrolled patients. Account information was emailed to the patients, and they were asked to complete a baseline questionnaire regarding medication adherence. Patients who did not complete a baseline questionnaire were reminded via email or text message, based on patient preferences. Patients were then asked to complete a questionnaire every 2-3 months after baseline. Reminders were sent to unresponsive participants. After successful completion of each questionnaire, patients were mailed a $\$ 10$ gift card to a local grocery store, gas station, or restaurant. 


\section{$3 \quad$ Results}

Primary care clinicians were eager to enroll in the study. Of the 20 total clinicians who practiced across the three clinic sites, $15(75 \%)$ consented to participate in the study. Reasons for not consenting included pending retirement $(\mathrm{N}=2)$, too busy $(\mathrm{N}=1)$, too few diabetic patients $(\mathrm{N}=1)$, and unknown $(\mathrm{N}=1)$. Clinicians largely perceived their diabetic populations as needing more attention with respect to medication adherence, so they elected to try out the clinical dashboard in their practice.

Patients were less eager to enroll and engage. Out of 906 patients approached for recruitment, only 203 (22\%) patients completed screening $(92 ; 10 \%$ refused screening). Of those who completed screening, 108 (53\%) were both eligible (defined as having access to a computer and able to provide informed consent) and interested in the study. Of those patients who did not participate, 72 (35\%) were found to be ineligible and $27(13 \%)$ refused to participate after the screening. Lack of routine access to a computer or the Internet was the primary reason ineligibility.

A total of 96 patients were enrolled. The research assistant was able to create accounts for 92 enrolled patients. By the end of the pilot, just 24 (26\%) patients successfully logged onto the portal and completed a questionnaire regarding their barriers to medication adherence. Many enrolled patients struggled due to computer or Internet access issues. For example, one patient repeatedly stated she was waiting for her daughter to come over to help her. Other patients' email addresses bounced less than 1-2 weeks after they provided them during the enrollment process. Text messaging and phone calls were helpful in reaching some of these patients, but access challenges remained for a significant number of study subjects.

\section{Discussion}

To fully realize the potential of health information technologies to impact population health outcomes, information and systems need to be integrated. Prior studies to improve adherence have focused on singular modalities to change provider or patient behavior. For example, in Vollmer et al [26] an interactive voice response system called patients who appeared to have gaps in refilling their asthma medication. The system was statistically significant in changing adherence, but the mean change was not clinically meaningful. Similarly, a recent systematic review of patient portals identified just one study that demonstrated an effect on diabetes care delivery [27]. However, in the one identified study, the portal was found to be associated with a change in medication regimen but had no impact on clinical outcomes as measured by HbA1c and blood pressure [28]. Our DM module integrates multiple information tools to address medication adherence in a coordinated fashion. While our approach may not be the only method to stimulate better adherence for patients with chronic illness, we believe that future approaches need to draw upon the power of EHR, PHR, and CDS systems to make adherence easier in the context of routine clinical care.

To be effective, 'routine clinical care' must include environments where patients exist (e.g., home, work, bus stop). Integrated informations solutions therefore must 
incorporate technologies that can be integrated into daily routines of people. Our efforts were hampered by system barriers that remain a challenge for many people, especially those who are elderly or of low socioeconomic status [29]. Therefore a digital divide still exists, even if it may be shrinking in populations burdened by multiple co-morbid diseases requiring complex drug regimens.

Finally, it is possible that patients may be resistant to describing barriers they experience to taking medications as prescribed by their providers. These may include embarrassment over non-compliance, their economic status, or failure to disclose secondary problems such as side effects. Resistance may also reflect a fear that disclosing issues may result in having to take additional medications. Furthermore, resistance could suggest there is simply a lack of interest in logging onto a portal to communicate. Future research will need to explore the factors that impact patients' willingness to use a portal to communicate with their providers about medication adherence.

Some of the challenges we describe, however, might be overcome with a smart phone app that integrates better into daily routine over an Internet-based app that must be used on a computer. Yet singular mobile apps that focus on just one aspect of health or wellness may not be usable in large populations. Multi-functional apps may be, or at least apps that cover multiple aspects of a chronic disease. For example, management of DM involves not only medication adherence but also changes to diet, exercise, and co-morbid conditions such as hypertension. Therefore technologies that engage patients will likely be those which can address multiple concerns in an integrated fashion (e.g., one-stop shop) rather than require multiple apps or interfaces. In addition, it will become critical that future applications include appropriate education that emphasizes the importance of patient-provider communication to enable shared decision making that will achieve optimal therapeutic outcomes.

\section{Conclusions}

We engaged a small number of physicians and patients in using novel e-health technologies to improve medication adherence by combining patient medication history data from a combination of electronic data sources with patient-reported barriers. Engagement of providers and patients was challenging. While providers were eager to enroll and try out the clinical dashboard, many patients experienced challenges in gaining routine access to the Internet to use a portal designed for patient engagement. Limited usage prevents integrated e-health technologies from impacting outcomes as providers continue to only see a partial picture of health. Further, digital divide issues remain important to address so that all patients can access and use e-health technologies, especially those who are vulnerable and at risk of serious complications from poor medication management.

Acknowledgements. This work was supported by a grant (R34DK092769) from the National Institute for Diabetes and Digestive and Kidney Diseases and by the Department of Veterans Affairs, Veterans Health Administration, Health Services 
Research and Development Service CIN 13-416. Dr. Dixon is a Health Research Scientist at the Richard L. Roudebush Veterans Affairs Medical Center in Indianapolis, Indiana, USA. Dr. Dixon also receives funding from a Robert Wood Johnson Foundation Mentored Research Scientist Development Award (71596). The content is solely the responsibility of the authors and does not necessarily represent the official views of the National Institutes of Diabetes and Digestive and Kidney Diseases, the National Institutes of Health, the Department of Veterans Affairs, the Robert Wood Johnson Foundation, or the United States Government.

\section{References}

1. George, B., Cebioglu, M., Yeghiazaryan, K.: Inadequate diabetic care: global figures cry for preventive measures and personalized treatment. The EPMA Journal 1, 13-18 (2010)

2. Kim, C., Williamson, D.F., Herman, W.H., Safford, M.M., Selby, J.V., Marrero, D.G., Curb, J.D., Thompson, T.J., Narayan, K., Mangione, C.M.: Referral management and the care of patients with diabetes: the Translating Research Into Action for Diabetes (TRIAD) study. The American Journal of Managed Care 10, 137 (2004)

3. Kim, C., Williamson, D.F., Mangione, C.M., Safford, M.M., Selby, J.V., Marrero, D.G., Curb, J.D., Thompson, T.J., Narayan, K.V., Herman, W.H.: Managed Care Organization and the Quality of Diabetes Care The Translating Research Into Action for Diabetes (TRIAD) study. Diabetes Care 27, 1529-1534 (2004)

4. Levit, K., Smith, C., Cowan, C., Lazenby, H., Sensenig, A., Catlin, A.: Trends in US health care spending, 2001. Health Affairs 22, 154-164 (2003)

5. Kerr, E.A., Gerzoff, R.B., Krein, S.L., Selby, J.V., Piette, J.D., Curb, J.D., Herman, W.H., Marrero, D.G., Narayan, K.V., Safford, M.M.: Diabetes care quality in the Veterans Affairs Health Care System and commercial managed care: the TRIAD study. Annals of Internal Medicine 141, 272-281 (2004)

6. Mangione, C.M., Gerzoff, R.B., Williamson, D.F., Steers, W.N., Kerr, E.A., Brown, A.F., Waitzfelder, B.E., Marrero, D.G., Dudley, R.A., Kim, C.: The association between quality of care and the intensity of diabetes disease management programs. Annals of Internal Medicine 145, 107-116 (2006)

7. Hanlon, J.T., Schmader, K.E., Ruby, C.M., Weinberger, M.: Suboptimal prescribing in older inpatients and outpatients. Journal of the American Geriatrics Society 49, 200-209 (2001)

8. Vik, S.A., Maxwell, C.J., Hogan, D.B.: Measurement, correlates, and health outcomes of medication adherence among seniors. The Annals of Pharmacotherapy 38, 303-312 (2004)

9. Benson, J., Britten, N.: Patients' decisions about whether or not to take antihypertensive drugs: qualitative study. BMJ 325, 873 (2002)

10. Cramer, J.A.: Microelectronic systems for monitoring and enhancing patient compliance with medication regimens. Drugs 49, 321-327 (1995)

11. Cramer, J.A.: Enhancing patient compliance in the elderly. Drugs \& Aging 12, 7-15 (1998)

12. Cramer, J.A.: A systematic review of adherence with medications for diabetes. Diabetes Care 27, 1218-1224 (2004)

13. Farmer, K.C.: Methods for measuring and monitoring medication regimen adherence in clinical trials and clinical practice. Clinical Therapeutics 21, 1074-1090 (1999) 
14. Brown, M.T., Bussell, J.K.: Medication adherence: WHO cares? Mayo Clin Proc. 86, 304$314(2011)$

15. Dixon, B.E., Jabour, A.M., Phillips, E.O., Marrero, D.G.: An informatics approach to medication adherence assessment and improvement using clinical, billing, and patient-entered data. J. Am. Med. Inform. Assoc. 21, 517-521 (2014)

16. McDonald, C.J., Overhage, J.M., Tierney, W.M., Dexter, P.R., Martin, D.K., Suico, J.G., Zafar, A., Schadow, G., Blevins, L., Glazener, T., Meeks-Johnson, J., Lemmon, L., Warvel, J., Porterfield, B., Cassidy, P., Lindbergh, D., Belsito, A., Tucker, M., Williams, B., Wodniak, C.: The Regenstrief Medical Record System: a quarter century experience. Int. J. Med. Inform. 54, 225-253 (1999)

17. Simonaitis, L., Belsito, A., Overhage, J.M.: Enhancing an ePrescribing system by adding medication histories and formularies: the Regenstrief Medication Hub. In: AMIA Annu. Symp. Proc., pp. 677-681 (2008)

18. Sikka, R., Xia, F., Aubert, R.E.: Estimating medication persistency using administrative claims data. The American Journal of Managed Care 11, 449-457 (2005)

19. Zhu, V.J., Tu, W., Rosenman, M.B., Overhage, J.M.: Facilitating Clinical Research through the Health Information Exchange: Lipid Control as an Example. In: AMIA Annu Symp Proc. 2010, pp. 947-951 (2010)

20. Mamlin, B.W., Biondich, P.G., Wolfe, B.A., Fraser, H., Jazayeri, D., Allen, C., Miranda, J., Tierney, W.M.: Cooking up an open source EMR for developing countries: OpenMRS a recipe for successful collaboration. In: AMIA Annu. Symp. Proc., pp. 529-533 (2006)

21. Mohammed-Rajput, N.A., Smith, D.C., Mamlin, B., Biondich, P., Doebbeling, B.N.: OpenMRS, a global medical records system collaborative: factors influencing successful implementation. In: AMIA Annu. Symp. Proc. 2011, pp. 960-968 (2011)

22. Monahan, P., Lane, K., Hayes, R., McHorney, C., Marrero, D.: Reliability and validity of an instrument for assessing patients' perceptions about medications for diabetes: the PAMD. Qual. Life Res. 18, 941-952 (2009)

23. Marrero, D., Monahan, P., Lane, K., Hayes, R.: Validation of a scale to measure patientperceived barriers to medication use. 2006 International Society for Quality of Life Research meeting abstracts. Qual. Life Res. 15, A34-A35, Abstract \#1223 (2006)

24. Tang, P.C., Ash, J.S., Bates, D.W., Overhage, J.M., Sands, D.Z.: Personal health records: definitions, benefits, and strategies for overcoming barriers to adoption. J. Am. Med. Inform. Assoc. 13, 121-126 (2006)

25. Yamin, C.K., Emani, S., Williams, D.H., Lipsitz, S.R., Karson, A.S., Wald, J.S., Bates, D.W.: The digital divide in adoption and use of a personal health record. Arch. Intern. Med. 171, 568-574 (2011)

26. Vollmer, W.M., Feldstein, A., Smith, D.H., Dubanoski, J.P., Waterbury, A., Schneider, J.L., Clark, S.A., Rand, C.: Use of health information technology to improve medication adherence. Am. J. Manag. Care 17, SP79-SP87 (2011)

27. Ammenwerth, E., Schnell-Inderst, P., Hoerbst, A.: The impact of electronic patient portals on patient care: a systematic review of controlled trials. Journal of Medical Internet Research 14 (2012)

28. Grant, R.W., Wald, J.S., Schnipper, J.L., Gandhi, T.K., Poon, E.G., Orav, E.J., Williams, D.H., Volk, L.A., Middleton, B.: Practice-linked online personal health records for type 2 diabetes mellitus: a randomized controlled trial. Archives of Internal Medicine 168, 1776 (2008)

29. Pew Research Center http: / / pewinternet.org/ /media//Files/Reports / 2013 /PIP_Offline\%20adults_092513_PDF.pdf 